\title{
Threatened Flora of Ilha Grande, Rio de Janeiro State, Brazil
}

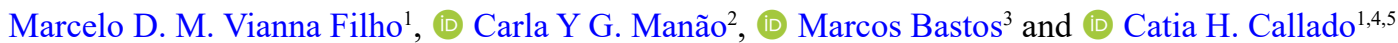

Received: 16.07.2019; accepted: 01.07.2020

How to cite: Vianna Filho, M.D.M., Manão, C.YG., Bastos, M. \& Callado, C.H. 2020. Threatened Flora of Ilha Grande, Rio de Janeiro State, Brazil. Hoehnea 47: e772019. http://dx.doi.org/10.1590/2236-8906-77/2019.

ABSTRACT - (Threatened Flora of Ilha Grande, Rio de Janeiro State, Brazil) This study documents the occurrence of threatened plant species on Ilha Grande, Rio de Janeiro State, Brazil. This survey acquired data from herbaria, living collections and field sampling. A total of 49 threatened species distributed among 27 families and 38 genera were recorded in the scientific collections, of which 43 are cited on official lists of threatened plant species for the State of Rio de Janeiro and Brazil. Twelve species recorded on the island are on an international list of endangered species, 30 on the national list for Brazil, and 11 on the State List of Rio de Janeiro. The IUCN Red List categories of Vulnerable and Endangered were the predominant categories for the plant species of Ilha Grande, with the main historical threat factors being deforestation and logging. Most of the threatened species of the island are arboreous. The families with the greatest number of threatened species are also among the richest in Dense Ombrophilous Forest: Melastomataceae, Myrtaceae, Meliaceae, Bromeliaceae and Orchidaceae. Four species registered on the island also appear on the list of endemic species in the State of Rio de Janeiro.

Keywords: Angra dos Reis, floristic survey, insular environment, IUCN, protected areas, Red List

RESUMO - (Flora Ameaçada da Ilha Grande, Estado do Rio de Janeiro, Brasil) Este estudo apresenta as plantas ameaçadas da Ilha Grande, Estado do Rio de Janeiro, Brasil. Esta pesquisa inventariou dados de herbários, coleções vivas e amostragem de campo. Um total de 49 espécies ameaçadas distribuídas entre 27 famílias e 38 gêneros foram registradas em coleções científicas, das quais 43 são citadas em listas oficiais de espécies ameaçadas de plantas do Estado do Rio de Janeiro e do Brasil. Doze espécies registradas na ilha estão na lista internacional de espécies ameaçadas de extinção, 30 na lista para o Brasil e 11 na lista do Estado do Rio de Janeiro. As categorias da IUCN Vulnerável e Em Perigo são as predominantes para as espécies da Ilha Grande, com os principais fatores históricos de ameaça sendo o desmatamento e a extração de madeira. A maioria das espécies ameaçadas da Ilha são arbóreas. As famílias com o maior número de espécies ameaçadas também estão entre as mais ricas da Floresta Ombrófila Densa: Melastomataceae, Myrtaceae, Meliaceae, Bromeliaceae e Orchidaceae. Quatro espécies registradas na Ilha também aparecem na lista de espécies endêmicas do Estado do Rio de Janeiro. Palavras-chave: Ambiente insular, Angra dos Reis, Áreas protegidas, Inventário florístico, IUCN, Lista Vermelha

\section{Introduction}

Brazil is the most botanically diverse country in the world (Forzza et al. 2012), with about $50 \%$ of its seed plants being endemic to the country (BFG 2015). These are just two of the reasons why Brazil was one of the signatories of the Convention on Biological Diversity (GSPC 2011).
This initiative requires the development of local checklists, which are fundamental to accomplishing the targets of the Global Strategy for Plant Conservation (GSPC). Lists of endangered species in particular help to guide political, conservation and scientific actions aimed at protecting biodiversity (Mittermeier \& Scarano 2013). The species included in the list of threatened species of Brazilian flora

1. Universidade do Estado do Rio de Janeiro, Parque Botânico do Ecomuseu Ilha Grande, Rua São Francisco Xavier, 524, Maracanã, 20550-013 Rio de Janeiro, RJ, Brasil

2. Universidade Federal do Rio de Janeiro, Herbário RFA, Departamento de Botânica, Centro de Ciências da Saúde, Avenida Carlos Chagas Filho, 373, Cidade Universitária, Ilha do Fundão, 21941-902 Rio de Janeiro, RJ, Brasil

3. Universidade do Estado do Rio de Janeiro, Faculdade de Oceanografia, Rua São Francisco Xavier 524, Maracanã, 20550-013 Rio de Janeiro, RJ, Brasil

4. Universidade do Estado do Rio de Janeiro, Instituto de Biologia Roberto Alcantara Gomes, Departamento de Biologia Vegetal, Rua São Francisco Xavier, 524, 20550-013 Rio de Janeiro, RJ, Brasil

5. Corresponding author: catia.callado@gmail.com 
(MMA), established under Ordinance 443 (MMA 2014), and in the list of threatened endemic species of the State of Rio de Janeiro, CONEMA 80 (CONEMA 2018), are examples of red lists of species that have subsequently been protected by law.

The Atlantic Forest of Brazil is one of the world's biodiversity hotspots (Mittermeier et al. 2011). Located within this hotspot is the continental island Ilha Grande, which, with 19,300 ha, is the largest island in Ilha Grande Bay and one of the largest islands on the Brazilian coast. Ilha Grande belongs to the municipality of Angra dos Reis of the State of Rio de Janeiro (Rocha 2018). Throughout the last century, Ilha Grande was marked by fishing activities and the establishment of prisons in the villages of Abraão and Dois Rios (Prado 2003). With a significant reduction in fishing activity since the 1970s, and with the closure of prisons in the 1990s, tourism became the most important economic activity of the island (Mello 1987, Prado 2003, Santiago et al. 2009).

The first studies focusing on plants of Ilha Grande were those of Maciel et al. (1984), Araujo \& Oliveira (1988), Almeida et al. (1998), and Oliveira (1999, 2002). More recent plant inventories (Callado et al. 2009, Nunes-Freitas 2009, Manão 2011, Pederneiras et al. 2012, Rosa et. al. 2016) have added new records to the flora of the island. Ilha Grande is a mosaic of several conservation units covered by secondary forest, but also functions to conserve significant habitats (Alho 2002).

Red lists are essential tools for environmental governance, and so they must be supported by documented information to assure that risk categories are appropriately employed and that the reliability of the assessments can be checked (IUCN 2011, Penedo et al. 2015). The flora of Ilha Grande contains 1143 plant species, including 25 that are under threat according to the most comprehensive botanical inventory of the island to date (Callado et al. 2009). Maurenza et al. (2018) published a checklist of all the conservation units of the State of Rio de Janeiro in a review of the previous checklists of Ilha Grande. Because of the richness of plant species in the Atlantic Forest and the advances of research carried out on Ilha Grande, the threat status of the flora of the island required reassessment. Thus, we compiled and summarized information on threatened plant species of Ilha Grande. The aim was to present an updated red list of flowering plants of Ilha Grande of the municipality of Angra dos Reis, State of Rio de Janeiro, and to document the occurrence of threatened species in order to strengthen the argument for preserving this priority area for conservation.

\section{Material and methods}

Plants were studied in their natural habitats and from herbaria vouchers. For the latter the authors visited herbaria with significant representations of specimens from Ilha Grande: HB, HBR, RFFP, R and RB). The field study and the collections of biological material in Parque Estadual da Ilha Grande (PEIG), Rio de Janeiro, Brazil were registered with, and authorized by, SISBIO-IBAMA (number 53321/2014 and INEA-RJ 006/2015). Living specimens were also included from the living collection of Parque Botânico do Ecomuseu Ilha Grande (PaB), located in Vila de Dois Rios, Ilha Grande, Rio de Janeiro, Brazil, with voucher samples being deposited in the herbarium of Universidade do Estado do Rio de Janeiro (HRJ). Life forms were based on Raunkiaer (1934), while conservation status and types of threat followed IUCN (2011).

A combination of databases - Brazilian Flora Checklist (BFG 2015), INCT Virtual Herbarium (INCT 2019), JABOT (JBRJ 2018), and Flora do Brasil 2010 (2019) - was used to prepare the checklist. Initially a filtered list of angiosperm species that occur on Ilha Grande was downloaded from the available databases. This initial list consisted of 4,966 records (infraspecific taxa were not included). Our survey included of all available vouchers cited by the databases. Vouchers with provenance of Ilha Grande and identified by specialists were prioritized, aided by information provided by the contributors to the Brazilian list (Martinelli \& Morais 2013).

The study considered species under some degree of threat in at least one of the latest red lists: IUCN (2011), MMA(2014) or CONEMA (2018). Consequently, Critically Endangered (CR), Endangered (EN) and Vulnerable (VU) categories were considered in this work, and are collectively referred to herein as 'threatened'. Globally threatened species were cited according to the IUCN (IUCN 2011), nationally threatened species followed the Brazilian Red List of Threatened Plant Species (Martinelli \& Morais 2013), while State threatened species followed the Red Book of Endemic Flora of the State of Rio de Janeiro (Martinelli et al. 2018), MMA (2014) and CONEMA (2018).

Both the national red list and the red list of the State of Rio de Janeiro received the value of law upon their publication, as does the guide Wanted: Endemic Flora of the State of Rio de Janeiro (Rosa et. al. 2018), published by CONEMA (2018). Global risk assessments are according to IUCN categories (IUCN 2011).

\section{Results and discussion}

Number of species - Of the 680 species of angiosperms listed for Ilha Grande, 60 are considered threatened to some degree. Vouchers were located for 49 of these species, distributed among 27 families and 38 genera, cited in the official lists of threatened plants species of the State of Rio de Janeiro and the country of Brazil (table 1).

The 11 other species were indicated by other inventories (table 2) and do not have related herbarium material, which should be sought out in future floristic inventories. It should be emphasized that vouchers provide a means for other 
Table 1. Threatened species registered for Ilha Grande, Angra dos Reis, Rio de Janeiro State, Brazil. Classification of global threat is based on IUCN (2011), national (Brazil) threat is based on Martinelli \& Moraes (2013) and State (Rio de Janeiro) threat for endemic plants is based on Martinelli et al. (2018). Categories - VU: vulnerable; EN: endangered; CR: critically endangered; *: Rare species according to Giulietti et al. (2009).

\begin{tabular}{|c|c|c|c|c|c|}
\hline Family & Species & Global & $\begin{array}{l}\text { Nation } \\
\text { (Brazil) }\end{array}$ & $\begin{array}{l}\text { State } \\
(\mathrm{RJ})\end{array}$ & Reference/Voucher \\
\hline Amaryllidaceae & $\begin{array}{l}\text { Hippeastrum striatum (Lam.) } \\
\text { Moore }\end{array}$ & - & EN & - & $\begin{array}{l}\text { L.C. Giordano } 267 \\
\text { (RB278616) }\end{array}$ \\
\hline Annonaceae & Duguetia pohliana Mart. & - & - & $\mathrm{EN}$ & $\begin{array}{l}\text { Silva, A.T. et al. s.n. } \\
\text { (RBR32929) }\end{array}$ \\
\hline Araceae & Anthurium luschnathianum Kunth & - & EN & EN & M. Nadruz 1165 (RB323263) \\
\hline Araliaceae & $\begin{array}{l}\text { Dendropanax heterophyllus } \\
\text { (Marchal) Frodin }\end{array}$ & - & EN & $\mathrm{CR}$ & s.col., s.n. (HRJ00011194) \\
\hline Arecaceae & Euterpe edulis Mart. & - & VU & - & $\begin{array}{l}\text { M.D.M.Vianna Filho et al. } \\
3002\end{array}$ \\
\hline Aristolochiaceae & Aristolochia odora Steud. & - & VU & - & Lírio, E.J. 1199 (RB645859) \\
\hline Asteraceae & Mikania argyreiae DC & - & VU & - & $\begin{array}{l}\text { S.B. Gonçalves et al. } 105 \\
\text { (HRJ1447) }\end{array}$ \\
\hline Begoniaceae & $\begin{array}{l}\text { Begonia curtii L.B.Sm. \& } \\
\text { B.G.Schub. }\end{array}$ & - & VU & - & $\begin{array}{l}\text { M.V.S. Alves } 360 \\
\text { (RB301764) }\end{array}$ \\
\hline Bignoniaceae & $\begin{array}{l}\text { Adenocalymma ubatubense Assis } \\
\text { \& Semir*(new occurrence to RJ) }\end{array}$ & - & $\mathrm{CR}$ & - & $\begin{array}{l}\text { D.N.S. Machado } 372 \\
\text { (RB761724) }\end{array}$ \\
\hline Bromeliaceae & Neoregelia hoehneana L.B.Sm. & - & EN & - & $\begin{array}{l}\text { A.F. Nunes-Freitas et al. } 62 \\
\text { (RBR44565) }\end{array}$ \\
\hline Bromeliaceae & Vriesea rubyae E.Pereira & - & $\mathrm{CR}$ & EN & R. Moura 973 (RB1194) \\
\hline Bromeliaceae & Wittrockia superba Lindm. & - & EN & - & $\begin{array}{l}\text { A.F. Nunes-Freitas et al. } 94 \\
\text { (RBR44225) }\end{array}$ \\
\hline Erythroxylaceae & Erythroxylum ovalifolium Peyr. & - & - & VU & $\begin{array}{l}\text { T.A.P. Gonçalves } 10 \\
\text { (RB412988) }\end{array}$ \\
\hline Fabaceae & Inga lanceifolia Benth. & EN & $\mathrm{LC}$ & - & $\begin{array}{l}\text { M.F. Castilhori et al. } 1424 \\
\text { (HB8014) }\end{array}$ \\
\hline Fabaceae & Inga sellowiana Benth. & EN & NT & - & $\begin{array}{l}\text { Dorothy Araujo } 6217 \\
\text { (RB371309) }\end{array}$ \\
\hline Lauraceae & $\begin{array}{l}\text { Beilschmiedia angustifolia } \\
\text { Kosterm. }\end{array}$ & - & - & EN & $\begin{array}{l}\text { C.Y'G. Manão et al. } 923 \\
\text { (HRJ12994) }\end{array}$ \\
\hline Lauraceae & $\begin{array}{l}\text { Urbanodendron bahiense (Miens.) } \\
\text { Rohwer }\end{array}$ & $\mathrm{EN}$ & VU & - & $\begin{array}{l}\text { S.J. Silva Neto et al. s.n. } \\
\text { (HRJ12853) }\end{array}$ \\
\hline Lecythidaceae & $\begin{array}{l}\text { Couratari pyramidata (Vell.) } \\
\text { Kunth* (new occurrence) }\end{array}$ & EN & EN & EN & $\begin{array}{l}\text { F. Pinheiro et al. } 809 \\
\text { (HB8743) }\end{array}$ \\
\hline Maranthaceae & Ischnosiphon ovatus Körn. & - & EN & - & s.col., s.n. (HRJ00011607) \\
\hline
\end{tabular}


Table 1 (continuation)

\begin{tabular}{|c|c|c|c|c|c|}
\hline Family & Species & Global & $\begin{array}{l}\text { Nation } \\
\text { (Brazil) }\end{array}$ & $\begin{array}{l}\text { State } \\
(\mathrm{RJ})\end{array}$ & Reference/Voucher \\
\hline Melastomataceae & Bertolonia valenteana Baumgratz & - & - & $\mathrm{CR}$ & H.C. Lima 5776 (RB360091) \\
\hline Melastomataceae & Meriania glazioviana Cogn. & - & - & EN & $\begin{array}{l}\text { A.A.M. de Barros } 1428 \\
\text { (RB489040) }\end{array}$ \\
\hline Melastomataceae & Meriania longipes Triana & - & - & EN & $\begin{array}{l}\text { L.G.C. Rigon } 1408 \\
\text { (RB658363) }\end{array}$ \\
\hline Melastomataceae & Miconia gigantea Cogn. & - & - & EN & A.T. Silvas n. (ESA120363) \\
\hline Melastomataceae & $\begin{array}{l}\text { Pleroma thereminianum (DC.) } \\
\text { Triana }\end{array}$ & - & NT & EN & F. Pinheiro 419 (RB533992) \\
\hline Meliaceae & $\begin{array}{l}\text { Cedrela odorata L. (new } \\
\text { occurrence) }\end{array}$ & VU & VU & - & s.col. s.n. (HRJ11586) \\
\hline Meliaceae & Trichilia casaretti C.DC. & VU & $\mathrm{LC}$ & - & s.col.s.n. (HRJ11441) \\
\hline Meliaceae & Trichilia silvatica C.DC. & VU & $\mathrm{LC}$ & - & $\begin{array}{l}\text { R.R. Oliveira et al. s.n. } \\
\text { (ESA120107) }\end{array}$ \\
\hline Monimiaceae & Mollinedia acutissima Perkins & - & - & VU & R.Ribeiro 1977 (RB427454) \\
\hline Monimiaceae & $\begin{array}{l}\text { Mollinedia glabra (Spreng.) } \\
\text { Perkins }\end{array}$ & VU & $\mathrm{LC}$ & - & $\begin{array}{l}\text { C.Y'.G. Manão et al. s.n. } \\
\text { (HRJ12790) }\end{array}$ \\
\hline Moraceae & Ficus cyclophylla (Miq.) Miq. & EN & VU & - & B.E. Diaz 1564 (RB657573) \\
\hline Myristicaceae & $\begin{array}{l}\text { Virola bicuhyba (Schott ex } \\
\text { Spreng.) Warb. }\end{array}$ & - & EN & - & $\begin{array}{l}\text { L.P.G. Rosa et al. s.n. } \\
\text { (HRJ12565) }\end{array}$ \\
\hline Myrtaceae & $\begin{array}{l}\text { Calyptranthes fusiformis M.I. } \\
\text { Kawas. }\end{array}$ & - & VU & - & $\begin{array}{l}\text { A.A.M. de Barros } 1327 \\
\text { (RFFP3723) }\end{array}$ \\
\hline Myrtaceae & Eugenia bunchosiifolia Nied. & - & VU & - & F. Pinheiro 748 (RB 388775) \\
\hline Myrtaceae & $\begin{array}{l}\text { Eugenia malacantha D.Legrand } \\
\text { (new occurrence) }\end{array}$ & - & EN & - & $\begin{array}{l}\text { A. Giaretta } 1491 \\
\text { (ESA120502) }\end{array}$ \\
\hline Myrtaceae & Eugenia prasina Huber & VU & $\mathrm{LC}$ & - & $\begin{array}{l}\text { R. Marquete } 1210 \\
\text { (RB327150) }\end{array}$ \\
\hline Myrtaceae & Plinia edulis (Vell.) Sobral & - & VU & - & $\begin{array}{l}\text { L.P.G. Rosa et al. s.n. } \\
\text { (HRJ00012711) }\end{array}$ \\
\hline Orchidaceae & $\begin{array}{l}\text { Acianthera subrotundifolia (Cogn.) } \\
\text { F.Barros \&V.T.Rodrigues }\end{array}$ & - & - & VU & M.R.A Braga 352 (RB4477) \\
\hline Orchidaceae & Cattleya guttata Cogn. & - & VU & - & M.R.A Braga 179 (RB4353) \\
\hline Orchidaceae & $\begin{array}{l}\text { Grandiphyllum hians (Lindl.) } \\
\text { Docha Neto }\end{array}$ & - & VU & - & Moraes, M. 640 (RB4334) \\
\hline
\end{tabular}


Table 1 (continuation)

\begin{tabular}{|c|c|c|c|c|c|}
\hline Family & Species & Global & $\begin{array}{l}\text { Nation } \\
\text { (Brazil) }\end{array}$ & $\begin{array}{l}\text { State } \\
(\mathrm{RJ})\end{array}$ & Reference/Voucher \\
\hline Orchidaceae & Octomeria alexandri Schltr. & - & EN & - & M.R.A Braga 225 (RB4399) \\
\hline Orchidaceae & Stelis palmeiraensis Barb.Rodr. & - & - & VU & $\begin{array}{l}\text { M.R.A Braga } 359 \\
\text { (RB506035) }\end{array}$ \\
\hline Proteaceae & Roupala sculpta Sleumer* & - & VU & - & $\begin{array}{l}\text { Silva, AT et al. s.n. } \\
\text { (RBR00032998) }\end{array}$ \\
\hline Rubiaceae & Melanopsidium nigrum Colla & - & VU & - & A. Lobão 464 (RB348966) \\
\hline Rubiaceae & Rudgea macrophylla Benth. * & - & $\mathrm{EN}$ & NT & R. Ribeiro 1905 (RB490068) \\
\hline Sapindaceae & Cupania concolor Radlk. & - & VU & - & $\begin{array}{l}\text { D.M. Silva et al. } 96 \\
\text { (HB8999) }\end{array}$ \\
\hline Sapindaceae & Cupania furfuracea Radlk. & - & VU & - & $\begin{array}{l}\text { I.E. Santo et al. } 1271 \\
\text { (HB7983) }\end{array}$ \\
\hline Sapotaceae & $\begin{array}{l}\text { Chrysophyllum splendens Spreng. } \\
\text { (new occurrence) }\end{array}$ & VU & NT & - & Silva, AT s.n. (RBR33015) \\
\hline Sapotaceae & Pradosia kuhlmannii Toledo & - & $\mathrm{EN}$ & - & s.col., s.n. (HRJ11413) \\
\hline Urticaceae & $\begin{array}{l}\text { Coussapoa curranii S.F.Blake } \\
\text { (new occurrence) }\end{array}$ & VU & NT & - & $\begin{array}{l}\text { M.D.M.Vianna Filho et al. } \\
3001 \text { (HRJ13007) }\end{array}$ \\
\hline
\end{tabular}

researchers to verify published taxonomic determinations and ensure that taxonomic works are reliable and reproducible (Culley 2013). In some cases, target species are rare in the wild, and new records of occurrence are difficult to obtain (Giulietti et al. 2009). Such insufficient quantity of samples makes it challenging to make precise taxonomic determinations (Knapp 2002, Penedo et al. 2015). Of the forty-nine species registered and vouchered for Ilha Grande, 12 are on the IUCN Red List (IUCN 2011), 23 on the Brazilian national list (Martinelli \& Moraes 2013), upon which the national red list of endangered species was based (MMA 2014), and 14 as endemic threatened species for the State of Rio de Janeiro (Martinelli et al.2018), which were legally recognized by CONEMA (2018).

Areas of occurrence - Most of the threatened species are Atlantic Forest endemics (82\%), while, others are distributed in both the Atlantic Forest and Cerrado (16\%); a single species, Cedrela odorata L., Meliaceae, is broadly distributed among the Amazon Forest, Atlantic Forest, Caatinga and Cerrado biomes (2\%) (figure 1). Cedrela odorata is a species of concern due to reductions in its populations due to harvesting for timber, which has been used by traditional populations of Ilha Grande for boat construction.
No species have been described as endemic to Ilha Grande until now. Two species of Bromeliaceae described for the island - Vriesea aff. bituminosa (Moura 2011) and Canistropis aff. microps - when legitimately described, can be considered endemic.

Categories of threat - The predominant threat category for the threatened species of Ilha Grande is Vulnerable (24 spp.), followed by Endangered (21 spp.) and Critically Endangered (4 spp.) (figure 2). No extinct species emerged from the survey.

The studied species were placed in different categories by the different assessments: IUCN (2011), MMA (2014) or CONEMA (2018) (figure 3).

Some threatened species of Ilha Grande were treated as rare by Giulietti (2009): Adenocalymna ubatubense Assis \& Semir (Bignoniaceae), Couratari pyramidata (Vell.) Kunth (Lecythidaceae) and Roupala sculpta Sleumer (Proteaceae). Although considered rare by Giulietti (2009), Solanum carautae Carvalho has not been categorized as a threatened species but should be considered relevant to conservation.

The frequency distribution of the threat categories found for the threatened plant species of the island confirms what was reported in the Red List of the State of Rio de Janeiro, but with the addition of six new records (table 1) to the 


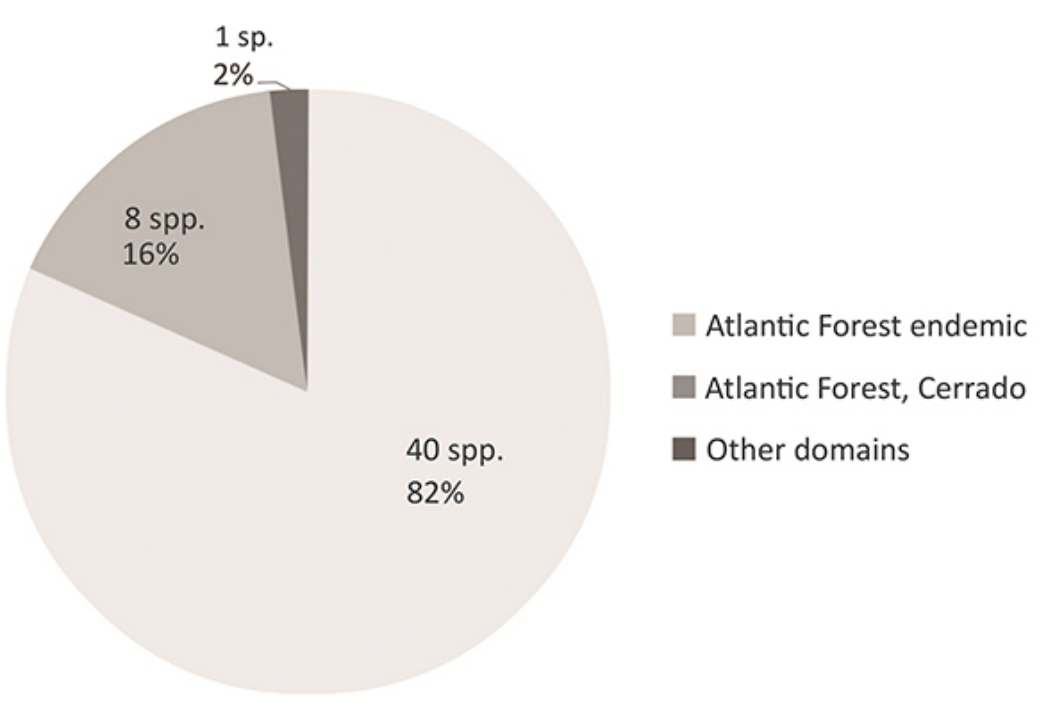

Figure 1. The number and percentage of threatened plant species, per biome of Ilha Grande, Angra dos Reis, Rio de Janeiro State, Brazil.

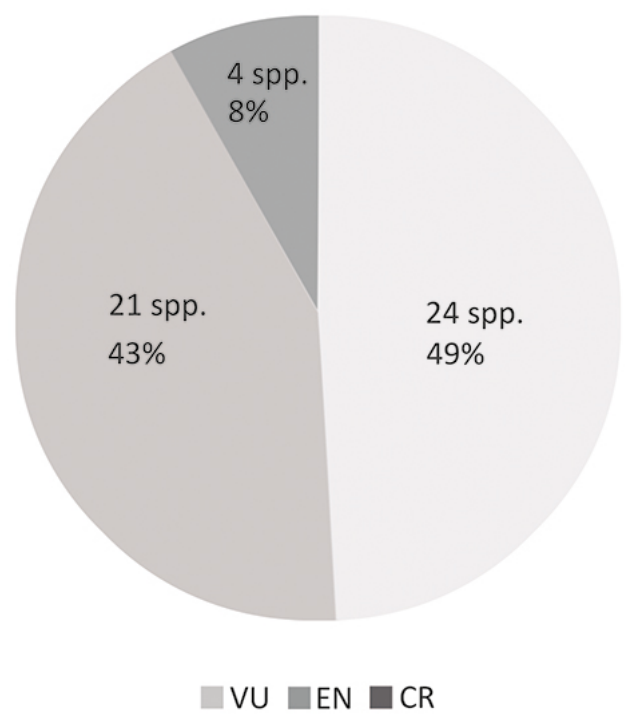

Figure 2. The number and percentage of threatened plant species, according to IUCN (2011) criteria, registered for Ilha Grande, Angra dos Reis, Rio de Janeiro State, Brazil. VU: Vulnerable; EN: Endangered; CR: Critically Endangered.

IUCN $(1998,2013)$

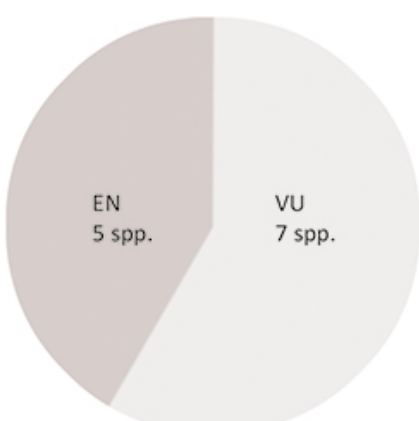

MMA (2014)

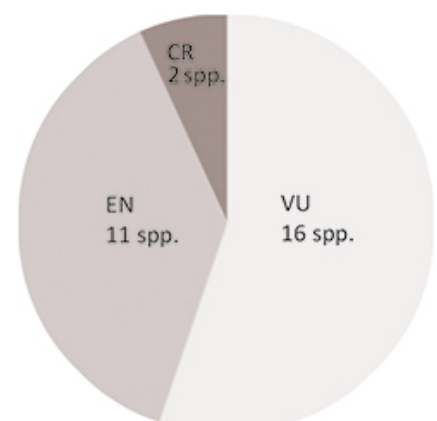

CONEMA (2018)

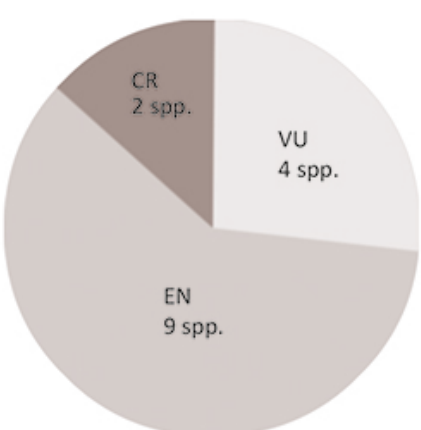

Figure 3. Distribution of threatened plant species, according to different assessment lists and following IUCN (2011) criteria, recorded for Ilha Grande, Angra dos Reis, Rio de Janeiro State, Brazil. VU: Vulnerable; EN: Endangered; CR: Critically Endangered. 
Table 2. Unvouchered threatened species indicated for Ilha Grande, Angra dos Reis, Rio de Janeiro State, Brazil. Classification of global threat is based on IUCN (2011), national (Brazil) threat is based on Martinelli \& Moraes (2013) and State (Rio de Janeiro) threat for endemic plants is based on Martinelli et al. (2018). Categories - VU: vulnerable; EN: endangered; CR: critically endangered.

\begin{tabular}{|c|c|c|c|c|c|}
\hline Family & Species & Global & $\begin{array}{l}\text { Nation } \\
\text { (Brazil) }\end{array}$ & $\begin{array}{l}\text { State } \\
(\mathrm{RJ})\end{array}$ & Reference/Voucher \\
\hline Begoniaceae & Begonia undulata Schott & - & EN & - & $\begin{array}{l}\text { Manão (2011) apud } \\
\text { Maurenza et al. (2018) }\end{array}$ \\
\hline Bignoniaceae & Tabebuia cassinoides (Lam.) DC & - & EN & - & $\operatorname{INEA}(2010)$ \\
\hline Chrysobalanaceae & Couepia schottii Fritch & VU & EN & - & Callado et al. (2009) \\
\hline Cyperaceae & $\begin{array}{l}\text { Pleurostachys pilulifera Longhi- } \\
\text { Wagner, Baldini \& A.C. Araújo }\end{array}$ & - & - & - & $\begin{array}{l}\text { Manão (2011) apud } \\
\text { Maurenza et al. (2018) }\end{array}$ \\
\hline Fabaceae & Inga platyptera Benth. & EN & VU & - & INEA (2010) \\
\hline Gesneriaceae & Codonanthe carnosa (Gardner) Hanst. & - & VU & - & Araujo e Oliveira (1988) \\
\hline Gesneriaceae & Sinningia pusilla (Mart.) Baill & - & - & EN & Callado et al. (2009) \\
\hline Meliaceae & Cedrela fissilis Vell. & EN & VU & - & Callado et al. (2009) \\
\hline Myrtaceae & Eugenia disperma Vell. & - & VU & - & $\operatorname{INEA}(2010)$ \\
\hline Myrtaceae & Eugenia vattimoana Mattos & - & VU & - & Callado et al. (2009) \\
\hline Orchidaceae & Rauhiella silvana Toscano & - & EN & - & Barros et al. (2015) \\
\hline
\end{tabular}

threatened flora of Ilha Grande by Maurenza et al. (2018). However, it was not possible to obtain vouchers for 11 of the species (table 2) recorded by Maurenza et al. (2018).

The proportion of threatened species (49) of all registered flowering plant species for the island (680) is $7.2 \%$, which is below the equivalent proportion of $43.8 \%$ for the entire State of Rio de Janeiro (Martinelli 2018). There are three hypotheses that can explain this low proportion: (1) the sampling effort has not yet reached its maximum, and there are still species to be documented; (2) the flora of Ilha Grande does not have the same environments and phytophysionomies as other areas of the State of Rio de Janeiro; and (3) the diversity of species of Ilha Grande is reduced due to the island effect (MacArthur \& Wilson 2001).

Taxonomic groups - The families with the greatest number of species under some degree of threat were Melastomataceae, Myrtaceae and Orchidaceae, with five species each, which together represented $30 \%$ of the threatened species of the island. These were followed by Bromeliaceae and Meliaceae with three species each and together representing $12 \%$ of the total (table 1). Over a third of the families (eight families, together representing $55 \%$ of the threatened species) are represented by only a single species (figure 4). Most species were trees, of which the families with the highest number of threatened species were also among the richest families in Dense Ombrophilous Forest: Melastomataceae (5), Myrtaceae(5) and Meliaceae (3). The second most speciose group according to habit was herbs, which was represented by the families Orchidaceae (6) and Bromeliaceae (3), which were also specious in the Dense Ombrophilous Forest, with some species also in Restinga vegetation near sea level (table 3).

In general, tree families, like Fabaceae, Myrtaceae, Lauraceae and Melastomataceae, receive more attention in floristic inventories, and thus there is a greater number of species of this habit recognized as threatened (Whitmore 1990, Lieberman 1996, Lomolino 2001). Some herbaceous threatened species occur near sea level in Restinga and in Dense Ombrophilous Forest along the trail to Pico do Papagaio at 800-1000 m.s.a.l. (table 3). This finding may reflect the fact that these regions are more accessible on the island (vs. sites without trails). 
Table 3. Threatened species registered for Ilha Grande, Angra dos Reis, Rio de Janeiro State, Brazil. Vegetative habits - Subsh: subshrub; Shrub: shrub; Tree: tree; Climb: climbing plants; Herb: herbaceous. Substrates - Epi: epiphyte; Rup: rupicolous; Hem: hemiepiphyte; Terr: terricolous. Phytogeographic domains - AF: Atlantic Forest; CE: Cerrado. Phytophysiognomies - CF: Ciliary Forest; DOF: Dense Ombrophylous Forest; Res: Restinga.

\begin{tabular}{|c|c|c|c|c|c|c|}
\hline Family & Species & Lifeform & Substrate & $\begin{array}{l}\text { Phytogeographic } \\
\text { domain }\end{array}$ & $\begin{array}{c}\text { Phytophysiog- } \\
\text { nomy }\end{array}$ & $\begin{array}{c}\text { Locality as voucher } \\
\text { label }\end{array}$ \\
\hline Amaryllidaceae & $\begin{array}{l}\text { Hippeastrum } \\
\text { striatum (Lam.) } \\
\text { Moore }\end{array}$ & Herb & Terr & $\mathrm{AF}$ & DOF, Res & $\begin{array}{l}\text { Caminho da Praia } \\
\text { de Saco das Palmas } \\
\text { para Lopes Mendes }\end{array}$ \\
\hline Annonaceae & $\begin{array}{l}\text { Duguetia pohliana } \\
\text { Mart. }\end{array}$ & Tree & Terr & $\mathrm{AF}$ & DOF & $\begin{array}{l}\text { PEIG, Unidade } \\
\text { amostral trilha } \\
\text { Enseada da Estrela } \\
380 / 2\end{array}$ \\
\hline Araceae & $\begin{array}{l}\text { Anthurium } \\
\text { luschnathianum } \\
\text { Kunth }\end{array}$ & Herb & Terr & $\mathrm{AF}$ & DOF & $\begin{array}{l}\text { Praia do Sul, } \\
\text { restinga, vertente } \\
\text { sul da Ilha }\end{array}$ \\
\hline Araliaceae & $\begin{array}{l}\text { Dendropanax } \\
\text { heterophyllus } \\
\text { (Marchal) Frodin }\end{array}$ & Shrub & Terr & $\mathrm{AF}$ & DOF & Ilha Grande \\
\hline Arecaceae & Euterpe edulis Mart. & Herb & Terr & $\mathrm{AF}, \mathrm{CE}$ & $\mathrm{CF}, \mathrm{DOF}$ & Cultivada no $\mathrm{PaB}$ \\
\hline Aristolochiaceae & $\begin{array}{l}\text { Aristolochia odora } \\
\text { Steud. }\end{array}$ & $\begin{array}{l}\text { Liana/ } \\
\text { climb. }\end{array}$ & Terr & $\mathrm{AF}$ & AA, DOF & $\begin{array}{l}\text { PEIG, trilha para o } \\
\text { Pico do Papagaio }\end{array}$ \\
\hline Asteraceae & $\begin{array}{l}\text { Mikania argyreiae } \\
\text { DC }\end{array}$ & $\begin{array}{l}\text { Liana/ } \\
\text { climb. }\end{array}$ & Terr & $\mathrm{AF}, \mathrm{CE}$ & AA, DOF & Ilha Grande \\
\hline Begoniaceae & $\begin{array}{l}\text { Begonia curtii } \\
\text { L.B.Sm. } \\
\text { \&B.G.Schub. }\end{array}$ & Subs & $\begin{array}{l}\text { Rup, } \\
\text { Terr }\end{array}$ & $\mathrm{AF}$ & DOF & $\begin{array}{l}\text { Reserva Biológica } \\
\text { da Praia do Sul. } \\
\text { Praia do Leste }\end{array}$ \\
\hline Bignoniaceae & $\begin{array}{l}\text { Adenocalymma } \\
\text { ubatubense Assis } \\
\text { \&Semir }\end{array}$ & $\begin{array}{l}\text { Liana/ } \\
\text { climb. }\end{array}$ & Terr & $\mathrm{AF}$ & DOF & $\begin{array}{l}\text { Vila dos Rios, } \\
\text { PEIG, acima da } \\
\text { Cachoeira da Mãe } \\
\text { d' Água, Represa, } \\
\text { Transect } 6\end{array}$ \\
\hline Bromeliaceae & $\begin{array}{l}\text { Neoregelia } \\
\text { hoehneana L.B.Sm. }\end{array}$ & Herb & Epi & $\mathrm{AF}$ & DOF & $\begin{array}{l}\text { Restinga da Praia } \\
\text { do Sul }\end{array}$ \\
\hline Bromeliaceae & $\begin{array}{l}\text { Vriesea rubyae } \\
\text { E.Pereira }\end{array}$ & Herb & Epi & $\mathrm{AF}$ & DOF & Pico do Papagaio \\
\hline Bromeliaceae & $\begin{array}{l}\text { Wittrockia superba } \\
\text { Lindm. }\end{array}$ & Herb & Epi, Rup & $\mathrm{AF}$ & DOF & Lagoa Verde, PEIG \\
\hline Erythroxylaceae & $\begin{array}{l}\text { Erythroxylum } \\
\text { ovalifolium Peyr. }\end{array}$ & Shrub & Terr & $\mathrm{AF}$ & Res & $\begin{array}{l}\text { Reserva Biológica } \\
\text { Estadual da Praia } \\
\text { do Sul. Transição } \\
\text { da restinga aberta } \\
\text { para mata de } \\
\text { restinga logo depois } \\
\text { da primeira clareira } \\
\text { na trilha para a } \\
\text { Lagoa do Sul }\end{array}$ \\
\hline Fabaceae & $\begin{array}{l}\text { Inga lanceifolia } \\
\text { Benth. }\end{array}$ & $\begin{array}{c}\text { Shrub, } \\
\text { Tree }\end{array}$ & Terr & $\mathrm{AF}$ & DOF & Ilha Grande \\
\hline
\end{tabular}


Table 3 (continuation)

\begin{tabular}{|c|c|c|c|c|c|c|}
\hline Family & Species & Lifeform & Substrate & $\begin{array}{l}\text { Phytogeographic } \\
\text { domain }\end{array}$ & $\begin{array}{l}\text { Phytophysiog- } \\
\text { nomy }\end{array}$ & $\begin{array}{c}\text { Locality as voucher } \\
\text { label }\end{array}$ \\
\hline Fabaceae & $\begin{array}{l}\text { Inga sellowiana } \\
\text { Benth. }\end{array}$ & $\begin{array}{l}\text { Shrub, } \\
\text { Tree }\end{array}$ & Terr & $\mathrm{AF}$ & DOF, Res & $\begin{array}{l}\text { Reserva Biológica } \\
\text { da Praia do Sul }\end{array}$ \\
\hline Lauraceae & $\begin{array}{l}\text { Beilschmiedia } \\
\text { angustifolia } \\
\text { Kosterm. }\end{array}$ & Tree & Terr & $\mathrm{AF}$ & DOF & $\begin{array}{l}\text { Dois Rios, depois } \\
\text { da Trilha da } \\
\text { Jararaca, próximo } \\
\text { ao segundo rio }\end{array}$ \\
\hline Lauraceae & $\begin{array}{l}\text { Urbanodendron } \\
\text { bahiense (Meisn.) } \\
\text { Rohwer }\end{array}$ & Tree & Terr & $\mathrm{AF}$ & DOF & Ilha Grande \\
\hline Lecythidaceae & $\begin{array}{l}\text { Couratari } \\
\text { pyramidata (Vell.) } \\
\text { Kunth }\end{array}$ & Tree & Terr & $\mathrm{AF}$ & DOF & Caxadaço \\
\hline Maranthaceae & $\begin{array}{l}\text { Ischnosiphon ovatus } \\
\text { Körn. }\end{array}$ & Herb & Terr & $\mathrm{AF}$ & DOF, Res & Ilha Grande \\
\hline Melastomataceae & $\begin{array}{l}\text { Bertolonia } \\
\text { valenteana } \\
\text { Baumgratz }\end{array}$ & Herb & Terr & $\mathrm{AF}$ & DOF & Pico do Papagaio \\
\hline Melastomataceae & $\begin{array}{l}\text { Meriania } \\
\text { glazioviana Cogn. }\end{array}$ & Tree & Terr & $\mathrm{AF}$ & DOF & $\begin{array}{l}\text { Vila Dois Rios, } \\
\text { Represa }\end{array}$ \\
\hline Melastomataceae & $\begin{array}{l}\text { Meriania longipes } \\
\text { Triana }\end{array}$ & Tree & Terr & $\mathrm{AF}$ & DOF & $\begin{array}{l}\text { E (573857m), } \\
\text { N (7471790m) }\end{array}$ \\
\hline Melastomataceae & $\begin{array}{l}\text { Miconia gigantea } \\
\text { Cogn. }\end{array}$ & Tree & Terr & $\mathrm{AF}$ & DOF & PEIG \\
\hline Melastomataceae & $\begin{array}{l}\text { Pleroma } \\
\text { thereminianum } \\
\text { (DC.) Triana }\end{array}$ & Shrub & $\begin{array}{l}\text { Rup, } \\
\text { Terr }\end{array}$ & $\mathrm{AF}$ & DOF & $\begin{array}{l}\text { Vila Dois Rios. } \\
\text { Mãe d'água }\end{array}$ \\
\hline Meliaceae & Cedrela odorata L. & Tree & Terr & $\mathrm{AM}, \mathrm{CA}, \mathrm{AF}, \mathrm{CE}$ & DOF & Ilha Grande \\
\hline Meliaceae & $\begin{array}{l}\text { Trichilia casaretti } \\
\text { C.DC. }\end{array}$ & Tree & Terr & $\mathrm{AF}, \mathrm{CE}$ & $\mathrm{CF}, \mathrm{DOF}$ & Ilha Grande \\
\hline Meliaceae & $\begin{array}{l}\text { Trichilia sylvatica } \\
\text { C.DC. }\end{array}$ & Shrub & Terr & $\mathrm{AF}, \mathrm{CE}$ & CF, DOF & $\begin{array}{l}\text { Trilha para o Pico } \\
\text { do Papagaio }\end{array}$ \\
\hline Monimiaceae & $\begin{array}{l}\text { Mollinedia } \\
\text { acutissima Perkins }\end{array}$ & Tree & Terr & $\mathrm{AF}$ & DOF & $\begin{array}{l}\text { Reserva Biológica } \\
\text { Estadual da Praia } \\
\text { do Sul }\end{array}$ \\
\hline Monimiaceae & $\begin{array}{l}\text { Mollinedia glabra } \\
\text { (Spreng.) Perkinsf }\end{array}$ & $\begin{array}{l}\text { Shrub, } \\
\text { Tree }\end{array}$ & Terr & $\mathrm{AF}$ & DOF, Res & Ilha Grande \\
\hline Moraceae & $\begin{array}{l}\text { Ficus cyclophylla } \\
\text { (Miq.) Miq. }\end{array}$ & Tree & $\begin{array}{l}\text { Hemi, } \\
\text { Terr }\end{array}$ & $\mathrm{AF}$ & DOF, Res & $\begin{array}{l}\text { Margens do mar no } \\
\text { local designado por } \\
\text { Lagoa Azul }\end{array}$ \\
\hline Myristicaceae & $\begin{array}{l}\text { Virola bicuhyba } \\
\text { (Schott ex Spreng.) } \\
\text { Warb. }\end{array}$ & Tree & Terr & $\mathrm{AF}$ & $\mathrm{CF}, \mathrm{DOF}$ & Ilha Grande \\
\hline Myrtaceae & $\begin{array}{l}\text { Calyptranthes } \\
\text { fusiformis M.I. } \\
\text { Kawas. }\end{array}$ & Tree & Terr & $\mathrm{AF}$ & DOF & $\begin{array}{l}\text { PEIG, Mata do } \\
\text { Caxadaço }\end{array}$ \\
\hline
\end{tabular}


Table 3 (continuation)

\begin{tabular}{|c|c|c|c|c|c|c|}
\hline Family & Species & Lifeform & Substrate & $\begin{array}{l}\text { Phytogeographic } \\
\text { domain }\end{array}$ & $\begin{array}{l}\text { Phytophysiog- } \\
\text { nomy }\end{array}$ & $\begin{array}{c}\text { Locality as voucher } \\
\text { label }\end{array}$ \\
\hline Myrtaceae & $\begin{array}{l}\text { Eugenia } \\
\text { bunchosiifolia } \text { Nied. }\end{array}$ & Tree & Terr & $\mathrm{AF}$ & DOF & Ilha Grande \\
\hline Myrtaceae & $\begin{array}{l}\text { Eugenia malacantha } \\
\text { D.Legrand }\end{array}$ & Tree & Terr & $\mathrm{AF}$ & DOF & $\begin{array}{l}\text { Trilha para o Pico } \\
\text { do Papagaio }\end{array}$ \\
\hline Myrtaceae & $\begin{array}{l}\text { Eugenia prasina } \\
\text { Huber }\end{array}$ & Tree & Terr & $\mathrm{AF}, \mathrm{CE}$ & DOF & $\begin{array}{l}\text { Trilha para o Pico } \\
\text { do Papagaio }\end{array}$ \\
\hline Myrtaceae & $\begin{array}{l}\text { Plinia edulis (Vell.) } \\
\text { Sobral }\end{array}$ & Tree & Terr & $\mathrm{AF}$ & DOF & Ilha Grande \\
\hline Orchidaceae & $\begin{array}{l}\text { Acianthera } \\
\text { subrotundifolia } \\
\text { (Cogn.) F.Barros \& } \\
\text { V.T.Rodrigues }\end{array}$ & Herb & Epi & $\mathrm{AF}$ & DOF & Ilha Grande \\
\hline Orchidaceae & $\begin{array}{l}\text { Cattleya guttata } \\
\text { Cogn. }\end{array}$ & Herb & Epi, Terr & $\mathrm{AF}$ & DOF, Res & $\begin{array}{l}\text { Restinga de Lopes } \\
\text { Mendes }\end{array}$ \\
\hline Orchidaceae & $\begin{array}{l}\text { Grandiphyllum } \\
\text { hians (Lindl.) } \\
\text { Docha Neto }\end{array}$ & Herb & Epi & $\mathrm{AF}$ & DOF & $\begin{array}{l}\text { Enseada do Abraão, } \\
\text { subida para o Pico } \\
\text { do Papagaio }\end{array}$ \\
\hline Orchidaceae & $\begin{array}{l}\text { Octomeria } \\
\text { alexandri Schltr. }\end{array}$ & Herb & Epi, Rup & $\mathrm{AF}, \mathrm{CE}$ & DOF & Ilha Grande \\
\hline Orchidaceae & $\begin{array}{l}\text { Stelis palmeiraensis } \\
\text { Barb.Rodr. }\end{array}$ & Herb & Epi & $\mathrm{AF}$ & DOF & $\begin{array}{l}\text { Em cultivo no JBRJ } \\
\text { n. } 4483\end{array}$ \\
\hline Proteaceae & $\begin{array}{l}\text { Roupala sculpta } \\
\text { Sleumer }\end{array}$ & Tree & Terr & $\mathrm{AF}$ & DOF & PEIG \\
\hline Rubiaceae & $\begin{array}{l}\text { Melanopsidium } \\
\text { nigrum Colla }\end{array}$ & $\begin{array}{l}\text { Shrub, } \\
\text { Tree }\end{array}$ & Terr & $\mathrm{AF}, \mathrm{CE}$ & Res & $\begin{array}{l}\text { Reserva Ecológica } \\
\text { Estadual da Praia } \\
\text { do Sul }\end{array}$ \\
\hline Rubiaceae & $\begin{array}{l}\text { Rudgea macrophylla } \\
\text { Benth. }\end{array}$ & $\begin{array}{l}\text { Shrub, } \\
\text { Tree }\end{array}$ & Terr & $\mathrm{AF}$ & DOF & $\begin{array}{l}\text { Reserva Biológica } \\
\text { Estacional da Praia } \\
\text { do Sul. }\end{array}$ \\
\hline Sapindaceae & $\begin{array}{l}\text { Cupania concolor } \\
\text { Radlk. }\end{array}$ & Tree & Terr & $\mathrm{AF}$ & DOF & $\begin{array}{l}\text { Mirante para Trilha } \\
\text { do Saco do Céu }\end{array}$ \\
\hline Sapindaceae & $\begin{array}{l}\text { Cupania furfuracea } \\
\text { Radlk. }\end{array}$ & Tree & Terr & $\mathrm{AF}$ & DOF & Ilha Grande \\
\hline Sapotaceae & $\begin{array}{l}\text { Chrysophyllum } \\
\text { splendens Spreng. }\end{array}$ & $\begin{array}{l}\text { Shrub, } \\
\text { Tree }\end{array}$ & Terr & $\mathrm{AF}$ & DOF & $\begin{array}{l}\text { Unidade amostral } \\
\text { trilha Enseada da } \\
\text { Estrela } 218 / 2\end{array}$ \\
\hline Sapotaceae & $\begin{array}{l}\text { Pradosia } \\
\text { kuhlmannii Toledo }\end{array}$ & Tree & Terr & $\mathrm{AF}$ & DOF & Ilha Grande \\
\hline Urticaceae & $\begin{array}{l}\text { Coussapoa curranii } \\
\text { S.F.Blake }\end{array}$ & Tree & $\begin{array}{l}\text { Hemi, } \\
\text { Terr }\end{array}$ & $\mathrm{AF}, \mathrm{CE}$ & DOF & $\begin{array}{l}\text { Matariz, trilha } \\
\text { para o mirante } \\
\text { do bananal. Mais } \\
\text { de um indivíduo } \\
\text { observado no } \\
\text { dossel }\end{array}$ \\
\hline
\end{tabular}




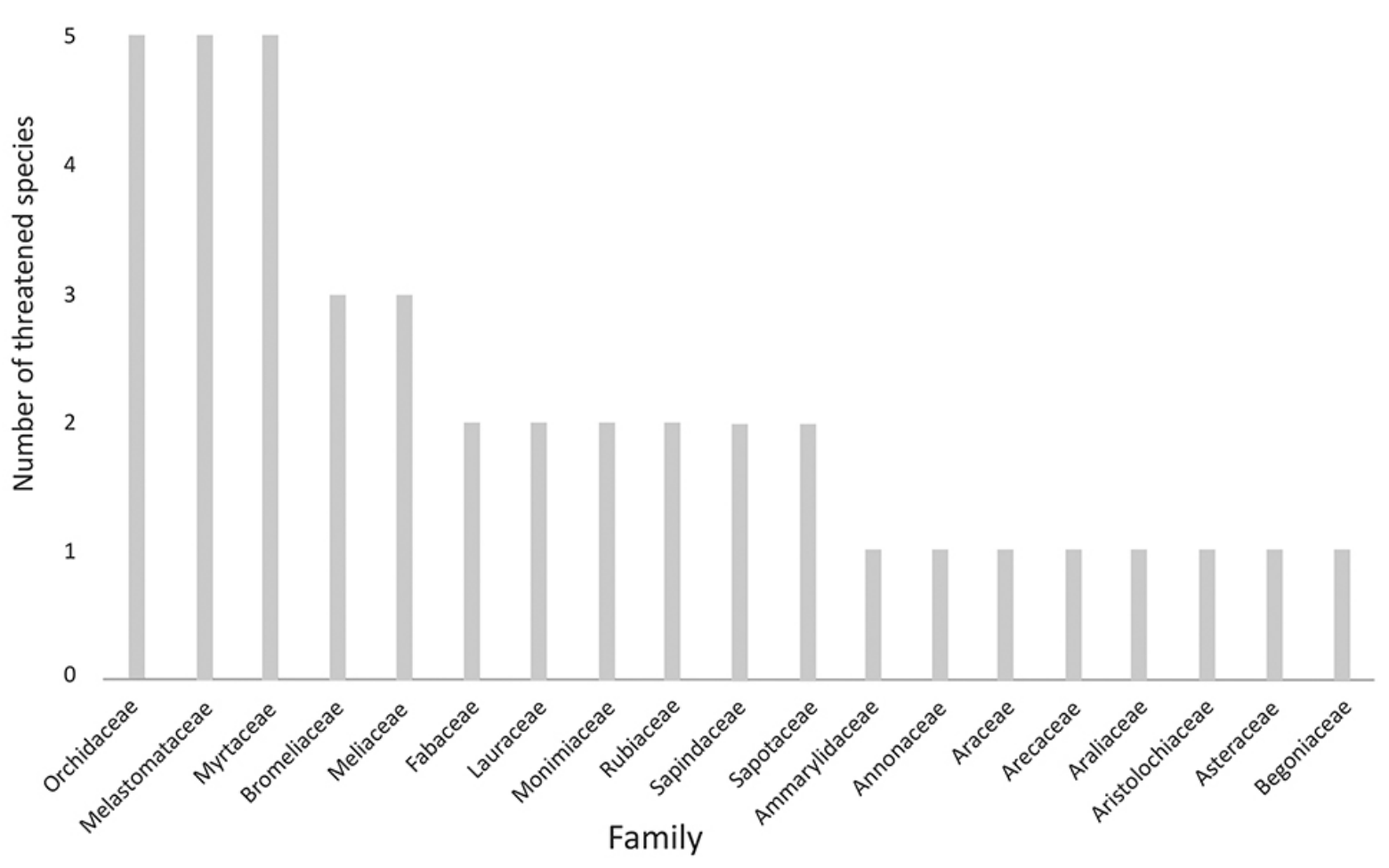

Figure 4. Distribution among botanical families of threatened plant species recorded for Ilha Grande, Angra dos Reis, Rio de Janeiro State, Brazil.

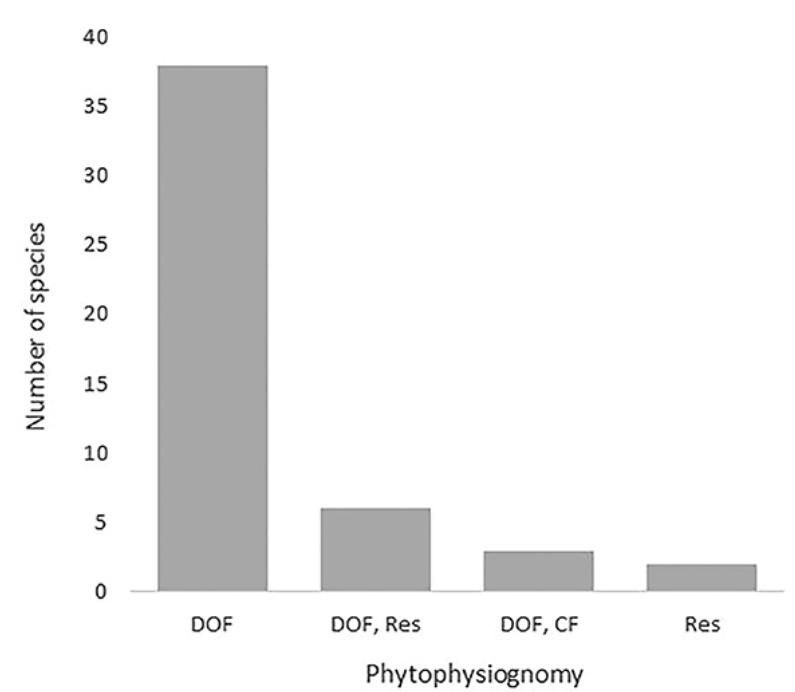

Figure 5. Distribution among phytophysiognomies of threatened species registered for Ilha Grande, Angra dos Reis, Rio de Janeiro State, Brazil. DOF: Dense Ombrophyllous Forest; Res: Restinga; CF: Ciliary Forest.

Of these families, Bromeliaceae, and Orchidaceae are important families, comprising epiphyte species (5 species, and 3 species), with some species occurring at elevations of up to $900 \mathrm{~m}$.

Occurrence in vegetation types - Dense Ombrophilous Forest was the phytophysionomy with the greatest richness of threatened species (figure 5). Some more speciose families in Dense Ombrophilous Forest with a high percentage of threatened species for the State of Rio de Janeiro (according to CONEMA 2018) do not have a proportional number of threatened species on the island (e.g., Bromeliaceae). This may be due to the fact that some species are epiphytic and more difficult to collect.

Most threatened species of Ilha Grande are terrestrial species (figure 6), although epiphytic species would be expected in areas of Atlantic Forest, especially in areas at higher elevations. It is believed that the low representation of epiphytes is due to a lack of collection effort since most inventories were based on terrestrial plant species (figure 7).

Threat factors influencing the conservation status of the flora of Ilha Grande - The main historical threat factors in Ilha Grande have been logging, deforestation for agriculture and surveillance, which were usual practices during the prison period, which ceased in 1994.

The predominant current threat types include deforestation and/or logging (table 1). Numerous areas throughout the island have become regenerated areas after abandonment in the 1990s. The primary areas of current impact are along the shoreline, especially in the surroundings of larger villages (Abraão, Araçatiba, Provetá), belonging to the conservation unit Area de Proteção Ambiental (APA) Tamoios. 
25

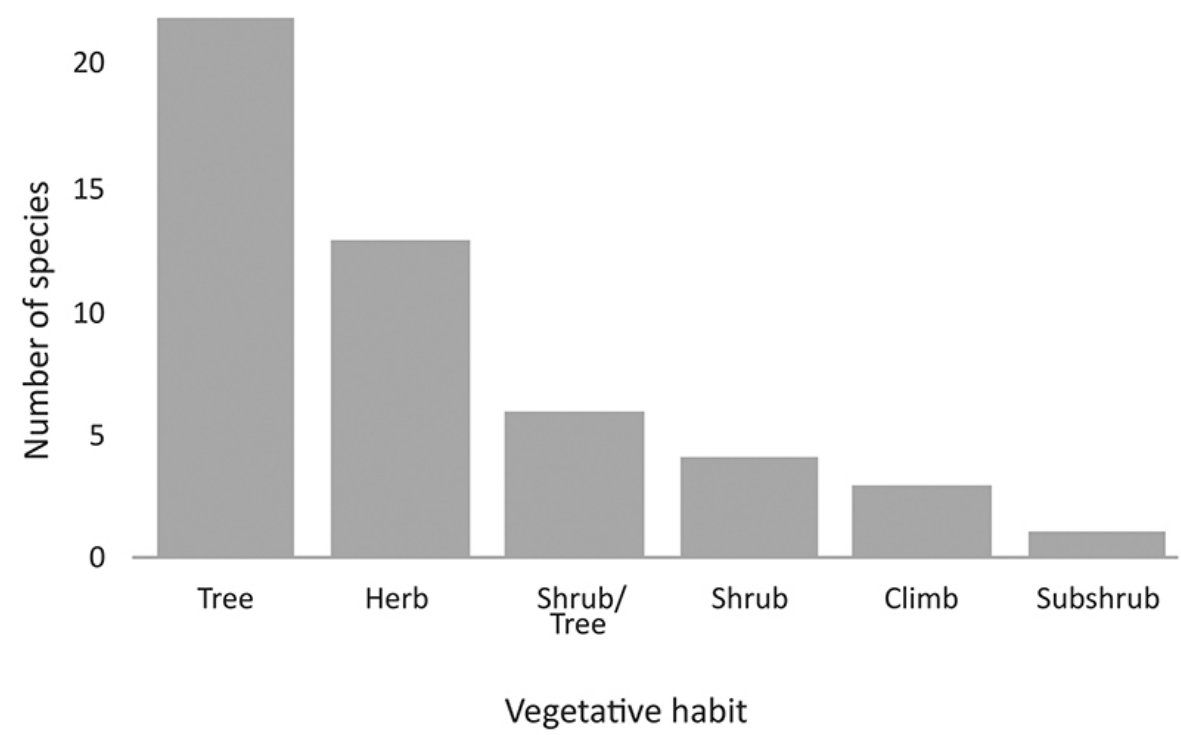

Figure 6. Distribution according to vegetative habit of threatened species registered for Ilha Grande, Angra dos Reis, Rio de Janeiro State, Brazil.

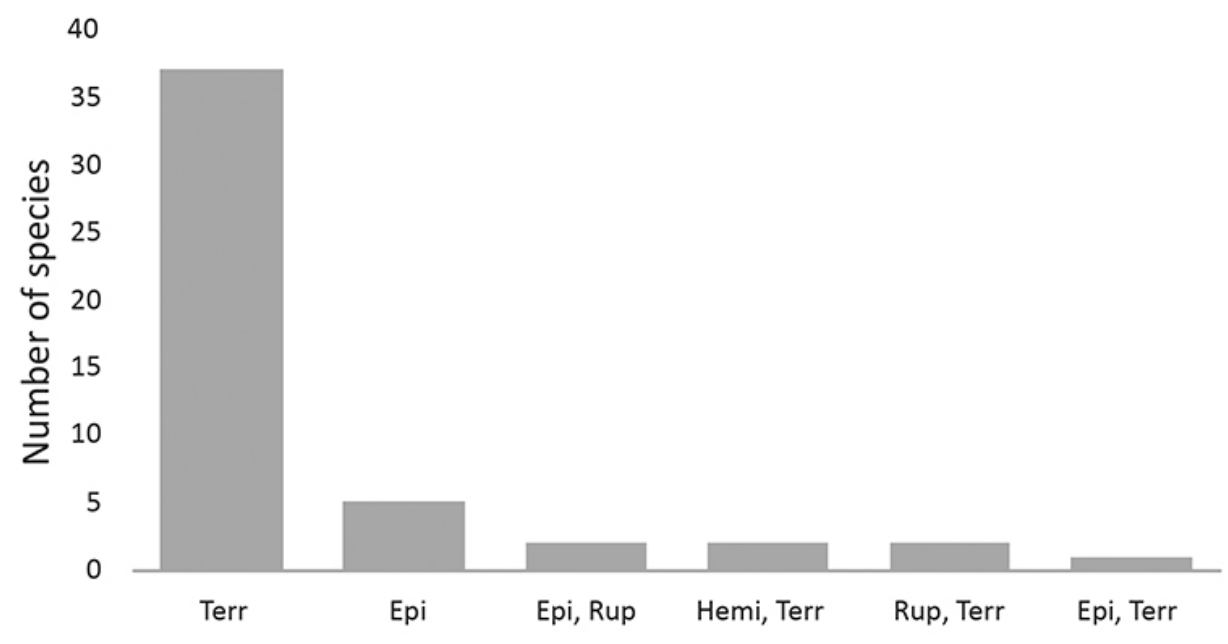

Life Form

Figure 7. Distribution according to life form (Raunkiaer 1932) of threatened species registered for Ilha Grande, Angra dos Reis, Rio de Janeiro State, Brazil. Terr: Terrestrial; Epi: Epiphytic; Rup: Rupicolous; Hemi: Hemi-epiphytic.

The presence of exotic species is also a potential problem for the maintenance of native species populations. Recent systematic surveys on different environments within the island recorded the occurrence of exotic species outside anthropic and peri-anthropic habitats (Callado et al. 2009). Invasive alien species have significant negative impacts on the recipient environment (Jeschke et al. 2014, Russell \& Blackburn 2017, Russel et al. 2017), primarily on biodiversity values and ecosystem services.
Collection efforts - The herbaria with the greatest holdings of botanical collections from Ilha Grande are: Jardim Botânico do Rio de Janeiro (RB), with $48 \%$ of the surveyed species, Universidade do Estado do Rio de Janeiro (HRJ, RFFP and HB), with 35\%, and Universidade Rural do Rio de Janeiro (RBR) with $12 \%$.

Rare and threatened plants are usually found in places that are difficult to access. Coincidentally or not, areas without access by road and steep access are the places with 
more rare species. These species are typically present in areas at middle/advanced (climax) stages of development.

Flagship species and species conservation - In conservation biology, a flagship species is a species selected to raise support for biodiversity conservation. Flagship species projects have sometimes been successful in saving species and their habitats (Barua 2011, Ducarne et al. 2011, Walpole 2002). Flagship species can be selected, depending on what is valued by the audience, and the goals of the project, such as conservation awareness, fundraising, ecotourism promotion, community-based conservation, and promotion of funded research (Barua 2011). Threatened species lists is therefore, an important indicator for new flagships species.

The most popular species among the endangered species of Ilha Grande is the palm jussara palm (Euterpe edulis Mart.). Exploitation of the heart of jussara palms is illegal (Brazil, 2008), but difficult to combat, and is in need of greater enforcement effort. The remaining populations, along the Abraão-Dois Rios road, serve as an active seedbank for seedling production, which is monitored by the Parque Botânico's staff.

Other species with appeal to become flagship species are the Bromeliad and Orchid species, as well as Couratari pyramidata (Vell.) Kunth (Lecythidaceae), a rare and singular tree, endemic to the Rio de Janeiro State.

\section{Conclusions}

Ilha Grande hosts 49 species that are under different degrees of threat at different geographic scales (global, national and state). These species reveal the importance of the island and its conservation units to the conservation of native plant diversity. Conservation programs that focus on endangered species are recommended, and will depend on scientific efforts in other scientific areas, as reproductive biology, demography, ecology, among others.

Fifteen species documented by previous studies for the island play a complementary role, for a potential total of 60 threatened species occurring on Ilha Grande (Araujo e Oliveira 1988, BFG 2015, Callado et al. 2009, INEA 2010, Manão 2011, Maurenza et al. 2018). Being unvouchered, these 11 species are not represented in herbarium collections (table 2), and so initial survey efforts to document these species by primary data (herbaria, photographs, and living collections) should spearhead future survey efforts. Due to the constant increase in knowledge about botanical species of the Atlantic Forest, species categorized as Near Threatened or in less severe categories may, in the future, be transferred to more severe categories of threat by upcoming assessments. With regard to long-term conservation of the populations of threatened species within protected areas of Ilha Grande, genetic exchanges with populations beyond the island are essential. Such exchanges require the maintenance of ecological corridors and mosaics of conservation units along the continental shoreline in Baía da Ilha Grande.

Conservation approaches focused on threatened species may benefit the entire biota of Baía da Ilha Grande, including the island itself and in its buffer area. There certainly remain gaps in the knowledge of the non-arborous flora of Ilha Grande, and so studies focused on less-studied groups should be encouraged for a more proper assessment of the threat level to this component of the biodiversity of Ilha Grande.

\section{Acknowledgements}

We are grateful to CEADS, ECOMIG and Programa de Pós graduação em Biologia Vegetal of Universidade do Estado do Rio de Janeiro (UERJ), for lab facilities and field support. We also thank Marcelo Fraga Castilhori and João Victor de Souza Castelar (UERJ), for assistance in the fieldwork. MDMVF received a grant from Coordenação de Aperfeiçoamento de Pessoal de Nível Superior - Brasil (CAPES) - Finance Code 001; CHC from Conselho Nacional de Desenvolvimento Científico e Tecnológico (CNPq); and CYGM from Fundação Carlos Chagas Filho para Pesquisa no Estado do Rio de Janeiro (FAPERJ). The field study and the collections of biological material in Parque Estadual da Ilha Grande (PEIG), Rio de Janeiro, Brazil were registered with, and authorized by, SISBIO-IBAMA (number 53321/2014 and INEA-RJ 006/2015) in a post-doctoral project (Process $\left.\mathrm{n}^{\mathrm{o}} 00 / 12469-3\right)$.

\section{Literature cited}

Alho, C.J.R., Schneider, M. \& Vasconcellos, L.A. 2002. Degree of threat to the biological diversity in the Ilha Grande State Park (RJ) and guidelines for conservation. Brazilian Journal of Biology 62(3): 375-385.

Almeida, D.R., Cogliatti-Carvalho, L. \& Rocha, C.F.D. 1998. As bromeliáceas da Mata Atlântica da Illha Grande, RJ: composição e diversidade de espécies em três ambientes diferentes. Revista Bromélia 5(1): 54-65.

Araujo, D.S.D. \& Oliveira, R.R. 1988. Reserva Biológica da Praia do Sul (Ilha Grande, Estado do Rio de Janeiro): lista preliminar da Flora. Acta Botanica Brasilica 1(2): 83-94.

Barua, M. 2011. Mobilizing metaphors: the popular use of keystone, flagship and umbrella species concepts. Biodiversity and Conservation 20(7): 1427-1440.

Barros, F. de, Vinhos, F., Rodrigues, V.T., Barberena, F.F.V.A., Fraga, C.N., Pessoa, E.M., Forster, W., Menini Neto, L., Furtado, S.G., Nardy, C., Azevedo, C.O., Guimarães, L.R.S. 2015. Orchidaceae in Lista de Espécies da Flora do Brasil. Jardim Botânico do Rio de Janeiro. Available at http://floradobrasil.jbrj.gov.br/ jabot/floradobrasil/FB12149 (access on 25-VII-2019). 
BFG - Brazilian Flora Group. 2015. Growing knowledge: an overview of Seed Plant. Rodriguésia 66(4): 10851113.

BRASIL. Ministério do Meio Ambiente. 2008. Instrução Normativa no 06, de 23 de setembro de 2008. Available at http:// www.mma.gov.br/estruturas/179/ arquivos/179_05122008033615.pdf. (access on 15VI-2019).

Callado, C.H., Barros, A.A.M., Ribas, L.A., Albarello, N., Gagliardi, R.F. \& Jascone, C.E. 2009. Flora e Cobertura Vegetal. In: Bastos, M.P. \& Callado C.H. (eds.). O ambiente da Ilha Grande. Rio de Janeiro: Centro de Estudos Ambientais e Desenvolvimento Sustentável, Rio de Janeiro, pp. 91-161.

CONEMA, Conselho Estadual de Meio Ambiente. 2018. Resolução CONEMA 80, de 24 de maio de 2018. Lista oficial das espécies de plantas endêmicas ameaçadas de extinção do estado do Rio de Janeiro - Diário Oficial do Estado do Rio de Janeiro, Rio de Janeiro.

Culley, T.M. 2013. Why vouchers matter in botanical research. Applied Plant Sciences 29(1): 11.

Ducarme, F., Luque, G.M. \& Courchamp, F. 2012. What are "charismatic species" for conservation biologists? BioSciences Master Reviews. (access on 13-VI-2019).

Flora do Brasil 2020 (em construção). 2019. Available at http://floradobrasil.jbrj.gov.br/ (access on 15-VI-2019).

Forzza, R.C., Baumgratz, J.F.A., Bicudo, C.E.M., Canhos, D., Carvalho Jr., A.A., Nadruz-Coelho, M.A. et al. 2012. New Brazilian floristic list highlights conservation challenges. BioScience 62: 39-45.

Giulietti, A.M., Rapini, A., Andrade, M.J.G., Queiroz, L.P. \& Silva, J.M.C. 2009. Plantas raras do Brasil. Belo Horizonte, Conservação Internacional, Belo Horizonte.

GSPC - Global Strategy for Plant Conservation. 2011. The Global Strategy for Plant Conservation: 20112020 - Botanic Gardens Conservation International, Richmond, UK. Available at https://www.plants2020. net/files/Plants2020/GSPCbrochure/gspc_english.pdf (access on 15-VI-2019).

INCT - Instituto Nacional de Ciência e Tecnologia. 2019. Herbario Virtual da Flora e dos Fungos - Species Link. Available at http://inct.splink.org.br/ (access on 15VI-2019).

INEA - Instituto Estadual do Ambiente. 2010. Plano de Manejo do Parque Estadual da Ilha Grande - ANEXOS. Available at https:// http://www.inea.rj.gov.br/wpcontent/uploads/2019/01/PEIG-PM-ANEXOS.pdf. (access on 15-VI-2019).

IUCN - International Union for Conservation of Nature. 2011. IUCN Red list categories and criteria: Version 3.1. IUCN Species Survival Commission. Available at https://www.iucnredlist.org/static/\%20categories_\%20 criteria_3_1 (access on 15-VI-2019).
JBRJ - Instituto de Pesquisas Jardim Botânico do Rio de Janeiro. 2018. Jabot - Banco de Dados da Flora Brasileira. Available at http://jabot.jbrj.gov.br/v2/ consulta.php (access on 2-XI-2018).

Jeschke, J.M., Bacher, S., Blackburn, T.M., Dick, J.T. \& Essl, F. 2014. Defining the impact of non-native species. Conservation Biology 28: 1188-1194.

Knapp, S. 2002. Solanum section Geminata (Solanaceae). Flora Neotropica Monograph 84: 1-404.

Lieberman, D., Liberman, M., Peralta, R. \& Hartshorn, G.S. 1996. Tropical forest structure and composition on a large-scale altitudinal gradient in Costa Rica. Journal of Ecology 84: 137-152.

Lomolino, M.V. 2001. Elevation gradients of speciesdensity: historical and prospective views. Global Ecology and Biogeography 10: 313.

MacArthur, R.H., \& Wilson, E.O. 2001. The theory of island biogeography. Princeton University Press. Princeton, New Jersey.

Maciel, N.C., Araujo, D.S.D. \& Magnanini, A. 1984. Reserva Biológica Estadual da Praia do Sul (Ilha Grande, Angra dos Reis, RJ). Contribuição para o conhecimento da Fauna e Flora. Bol. FBCN 19: 126-148.

Manão, C.Y.G. 2011. Composição Florística e Estrutura da comunidade arbustivo-arbórea de um trecho de floresta submontana na vertente sudeste do Parque Estadual da Ilha Grande, Angra dos Reis/RJ, MSc dissertation, Universidade do Estado do Rio de Janeiro, Rio de Janeiro.

Martinelli, G. \& Moraes, M.A. 2013. Livro Vermelho da Flora do Brasil. 1rd ed. Andrea Jakobsson Estúdio, Rio de Janeiro.

Martinelli, G., Martins, E., Moraes, M., Loyola, R. \& Amaro, R. 2018. Livro Vermelho da Flora Endêmica do Estado do Rio de Janeiro. Instituto de Pesquisas Jardim Botânico do Rio de Janeiro: Andrea Jakobsson, Rio de Janeiro.

Maurenza, D., Bocayuva, M., Pougy, N., Martins, E. \& Martinelli, G. 2018. Lista da Flora das Unidades de Conservação Estaduais do estado do Rio de Janeiro. Instituto de Pesquisas Jardim Botânico do Rio de Janeiro: Andrea Jakobsson, Rio de Janeiro.

Mello, C.E. 1987. Apontamentos para servir à história fluminense (Ilha Grande) Angra dos Reis. Angra dos Reis: Conselho Municipal de Cultura.

Mittermeier, C.G., Turner, W.R., Larsen, F.W., Brooks, T.M. \& Gascon, C. 2011. Global biodiversity conservation: the critical role of hotspots. In: Zachos, F.E. \& Habel, J.C. (eds.), Biodiversity Hotspots: Distribution and Protection of Priority Conservation Areas. SpringerVerlag, Berlin, pp. 3-22. 
Mittermeier, R. \& Scarano, F.R. 2013. Ameaças globais à biodiversidade de plantas. In: G. Martinelli, G. \& Moraes, M.A. (eds.) Livro vermelho da flora do Brasil. Rio de Janeiro: Instituto de Pesquisas Jardim Botânico do Rio de Janeiro, pp. 20-23.

MMA -Ministério do Meio Ambiente. 2014. Portaria No 443 de 17 de dezembro de 2014 - lista nacional oficial de espécies da flora ameaçadas de extinção. Diário Oficial da União - seção 1, n. 245, 18-XII-2014, pp. 110-121.

Moura, R.L. 2011. Revisão taxonômica do grupo Vriesea platynema Gaudich. (Bromeliaceae), PhD Thesis. Museu Nacional - Universidade Federal do Rio de Janeiro, Rio de Janeiro.

Nunes-Freitas, A.F., Rocha-Pessôa, T.C, Santos, A., Valente, C. \& Rocha-Duarte, C.F. 2009. Bromeliaceae da Ilha Grande, RJ: revisão da lista de espécies. Biota Neotropica 9(2): 213-219.

Oliveira, R.R. 1999. Levantamento florístico das Matas de Encosta da Vila Dois Rios, Ilha Grande, RJ. PhD Thesis, Universidade Federal do Rio de Janeiro, Rio de Janeiro.

Oliveira, R.R. 2002. Ação antrópica e resultantes sobre a estrutura e composição da Mata Atlântica na Ilha Grande, RJ. Rodriguésia 53: 33-58.

Pederneiras, L.C., Machado, A.F.P., Vianna Filho, M.D.M. \& Carrijo, T.T. 2012. Moraceae da Ilha Grande, Angra dos Reis Rio de Janeiro, Brasil. Revista do Instituto Florestal 24 (1): 21-33.

Penedo, T.S.A, Moraes, M.A., Borges, R.A.X., Maurenza, D.J., Diogo, M., \& Martinelli, G. 2015. Considerations on extinct species of Brazilian flora. Rodriguésia 66(3): 711-715. https: dx.doi.org/10.1590/2175-7860201566304

Prado, R. 2003. As espécies exóticas somos nós: Reflexão a propósito do Ecoturismo na Ilha Grande. Horizontes Antropológicos, Porto Alegre, ano 9, n. 20.
Raunkiaer, C.1934. The life forms of plants and statistical plant geography. Clarendon Press, Oxford.

Rocha, C.F.D., Telles-Silva, F.B., Vrcibradic, D. \& Nogueira-Costa, P. 2018. The Herpetofauna from Ilha Grande (Angra dos Reis, Rio de Janeiro, Brazil): updating species composition, richness, distribution and endemisms. Papéis Avulsos de Zoologia 58.

Rosa, L.P., Baumgratz, J.F.A. \& Silva-Neto, S.J. 2016. Miconia gigantea, a long-forgotten endemic and endangered species of Melastomataceae in the Brazilian Atlantic Forest. Anais da Academia Brasileira de Ciências 88: 1809-1818.

Rosa, P., Baez, C., Martins, E. \& Martinelli, G. 2018. Guia Procura-se: flora endêmica do estado do Rio de Janeiro. Instituto de Pesquisas Jardim Botânico do Rio de Janeiro: Graficci, Rio de Janeiro.

Russell, J.C. \& Blackburn, T.M. 2017. Invasive alien species: denialism, disagreement, definitions and dialogue. Trends in Ecology \& Evolution 32: 312-314.

Russell, J.C., Meyer, J.Y., Holmes, N.D. \& Pagad, N. 2017. Invasive alien species on islands: impacts, distribution, interactions and management. Available at https://www. researchgate.net/publication/317556219 (access 7-XI2019).

Santiago, A.M.A., Guimarães, C., Nogueira, I., Santos, M.S. dos \&Sant'Anna, T. 2009. História da Ilha Grande e patrimônio cultural. In: Bastos M. \& Callado, C.H. (Orgs.). O Ambiente da Ilha Grande, CEADS/UERJ, Rio de Janeiro. Cap. 8. pp. 299-369.

Walpole, M.J. \& Leader-Williams, N. 2002. Tourism and Flagship Species in Conservation. Biodiversity and Conservation. 11(3): 543-547.

Whitmore, T.C. 1990. An Introduction to Tropical Rain Forests. USA: Oxford University Press. 